\title{
Dinámica metropolitana en México
}

\author{
JORGE ISAURO RIONDA-RAMÍREZ*
}

\begin{abstract}
Nowadays, city growth is a very important subject given the evident change in pattern not only in demography but also in urban growth and development. Some of the problems and elements that have to be taken into account in the current social research programmes in the country include: Medium-sized cities, the accentuated precariousness of the country-side, the fast urban industrial ascent with the imminent tertiarisation of urban areas, the metropolisation of the largest cities in the country together with the phenomena of conurbation, satellite and peripheral development, industrial deconcentration and above all marginality. This paper denotes the character and some of the most important characteristics of these postmodern phenomena in the case of Mexico and in particular in regional development matters.
\end{abstract}

Keywords: metropolitan development, economic tertiarisation, marginality, urban development, industrial development.

\section{Resumen}

Actualmente, el crecimiento de las ciudades es tema relevante ante el evidente cambio de patrones tanto en la demografía como en el crecimiento y desarrollo urbanos. Las ciudades medias, la acentuada precarización del campo, el rápido ascenso industrial urbano y la inminente terciarización de las áreas urbanas, la metropolización de las grandes ciudades así como los fenómenos de conurbación, desarrollo satelital y periférico, la desconcentración industrial y, sobre todo, la marginación, son elementos que no deben dejarse de lado en los programas de investigación social del país. El presente trabajo denota el carácter y algunas de las características más sobresalientes de este fenómeno para el caso de México y, en especial, en materia de desarrollo regional.

Palabras clave: desarrollo metropolitano, terciarización económica, marginación, desarrollo urbano, desarrollo industrial.

* Universidad de Guanajuato, México. Correo-e: rionda@sicbasa.com 


\section{Planteamiento del problema}

En la fase de la producción flexible surge una nueva división y especialización internacional del trabajo. La terciarización de las economías a una escala mundial es un proceso irreversible y cada vez más vivencial en todos los ámbitos de la economía global.

En este proceso de especialización regional, los mercados de trabajo y sus estructuras observan procesos propios de la lógica de la producción flexible, por lo que su estudio es un tema de especial interés dentro de las ciencias sociales hoy día. El problema que se deriva es que no obstante el alto grado de desarrollo metropolitano, la pobreza y la marginación aumentan para un muy significativo estrato laboral.

\section{Supuesto}

La dinámica metropolitana en México es un fenómeno que se inscribe en la nueva lógica de acumulación capitalista, como parte de un capitalismo dual donde existe un centro hegemónico y una periferia dependiente, cuyo rol en la integración al esquema globalizador es un receptor de fuertes inversiones del centro que se canalizan a la producción de ciertas fases de la producción en un esquema mundialmente localizado. Los nexos que los negocios guardan con el nuevo esquema de producción flexible a escala transnacional generan nuevos patrones de crecimiento y desarrollo urbanos, en especial en materia de metropolización. Por ello la metropolización propia del posfordismo presenta su propia particularidad.

\section{Objetivo del trabajo}

En el presente artículo se trata de identificar los principales rasgos de la metropolización reciente en México y su nexo con el desarrollo de innovadores esquemas de producción flexible que se articulan en una escala internacional y que, necesariamente, dejan su huella en el desarrollo urbano metropolitano.

\section{Desarrollo}

Michael Janoschka esboza el marco teórico respectivo a los estudios urbanos y metropolitanos de las ciudades latinoamericanas e indica que: 
... desde los primeros bosquejos de una generalización esquemática de la metrópolis latinoamericana (Bähr, 1976) [...] las características de las urbes en América Latina se han visto fuertemente transformadas. Tanto los citados modelos como sus posteriores modificaciones y revisiones (Mertins, 1980 y 1995; Bähr y Mertins, 1981 y 1995; Borsdorf, 1982 y 1994) no relevan las nuevas tendencias de estructuración metropolitana. Por ejemplo, se han dado nuevos desarrollos urbano-espaciales que cambiaron la escala geográfica de la segregación socio-territorial, y al mismo tiempo, disminuyeron las diferencias de polarización entre la ciudad rica y la ciudad pobre. A gran escala se puede destacar un proceso de mezcla social, mientras a nivel micro se refuerza el patrón segregatorio (Sabatini, Cáceres y Cerda, 2001; Borsdorf, Bähr y Janoschka, 2002). Este principio de fragmentación territorial también determina la dispersión de infraestructura y funciones urbanas. Por ejemplo, los shoppings -en su concepción una copia de los malls norteamericanos- perdieron su cercanía espacial con las áreas de vivienda de las clases pudientes (Janoschka, 2002).

Por otra parte, es interesante ver cómo la sociología del trabajo revela un nuevo espectro no sólo en el ascenso urbano industrial de las naciones de reciente industrialización como México, sino del fenómeno metropolitano y sus mercados de trabajo, cuyos rasgos y patrones posmodernos observan nuevas contradicciones y otras anteriores más profundas. La polarización de los mercados conlleva fuertes nexos con el desarrollo metropolitano actual, especialmente el desarrollo de los mercados en la lógica del régimen de producción flexible.

Resalta la creciente polarización de los mercados de trabajo, sobre todo en el medio metropolitano. Esa tendencia se observa durante las décadas comprendidas entre 1970 y 1990 . El sector de servicios muestra la polarización de la estructura ocupacional entre estratos laborales de mayor calificación respecto de los menos calificados en actividades de tipo informal y polivalente. A raíz de los patrones observados en dichas décadas, los estudios actuales enfocan sus esfuerzos en comprender, dentro de la metropolización y el propio desarrollo urbano, la terciarización, fenómeno que es primordialmente urbano.

No obstante, como señala Sassen, el estudio de casos metropolitanos respecto de los mercados de trabajo debe considerar la especialización laboral con base en los procesos de producción de las empresas. La localización de estos procesos y la necesidad de trabajo especializado en las distintas esferas de la producción por fuerza tienen relación con la localización territorial de los negocios. Dice Sassen: 
Pero para los procesos de trabajo que requieren múltiples insumos especializados y una considerable innovación y riesgo, la necesidad de una interacción directa con otras empresas y especialistas se mantiene como un factor clave de localización. En consecuencia, la metropolización y regionalización de un sector económico tienen límites que se disponen en función del tiempo razonable de traslado a una ciudad o ciudades principales en la región (Sassen, 1998).

La región es necesariamente el marco donde se inscriben las consideraciones que se puedan hacer respecto a cómo comprendemos la metropolización. Para el caso de México, las distintas regiones que componen el territorio le dan un carácter distinto a la metropolización y por ello le otorgan también características diferentes. Especialmente si se habla del mercado de trabajo. A esta consideración no escapa la terciarización de la economía urbana y metropolitana.

La terciarización de la economía se da en dos ramales: el primero se especializa en los servicios a la producción, cuyos empleos están mejor remunerados y tienen un mayor estatus social, de tipo formal y crecen de forma significativa como negocios; el segundo atiende los servicios al consumo, es de bajo nivel de capitalización y remunerativo, de tipo informal y estatus social bajo, su crecimiento como negocio es modesto, no obstante, su presencia en el mercado crece de manera significativa principalmente como parte del comercio ambulante.

El sector terciario lleva en su seno las contradicciones inherentes al capitalismo, por lo que muestra graves contrastes y desigualdades en materia de desarrollo social, al igual que la organización del territorio expresa grandes contrastes entre áreas de alto dinamismo económico y regiones con grave rezago en su desarrollo.

El interés de estudios recientes radica en conocer la evolución diferenciada entre los servicios al productor y los servicios al consumo. Los primeros muestran un alto grado de concentración en las áreas metropolitanas densamente pobladas, donde los servicios están mejor remunerados y calificados en su mercado de trabajo. Los segundos por el contrario, observan una tendencia a la precarización de la mayor parte de su masa laboral.

Sassen sostiene que la concentración de los servicios se circunscribe cada vez más a algunas ciudades o metrópolis independientemente del lugar donde se concentren las actividades industriales. Por tradición, en las áreas metropolitanas se concentran las actividades industriales secundarias o las actividades 
de transformación, por lo que los servicios al productor tienden a concentrarse en ellas.

Hay un sector altamente internacionalizado que prospera. El desarrollo local poco a poco se orienta a los mercados foráneos, donde se ve el fuerte desarrollo de empresas maquiladoras transnacionales.

La población económicamente activa (PEA) disminuye en el sector industrial y aumenta continuamente en el sector servicios. En las áreas urbanas metropolitanas la industria observa una desconcentración, pues se sale de la mancha urbana para localizarse en áreas periféricas y satelitales, donde se asienta como distritos o parques industriales.

La industria muestra una contradicción entre sus sectores tradicionales compuestos por empresas familiares, micro y medianas, respecto del sector moderno donde destacan las grandes empresas, incluso el sector maquilador como parte de un nuevo estilo de industrialización posmoderna contribuye en esta dicotomía.

La ciudad, el medio urbano y las zonas metropolitanas son elementos más fehacientes del desarrollo globalizador, mientras que la precarización del medio rural se mantiene desde los años cuarenta en que la nación mexicana inicia su ascenso industrial urbano propio del modernismo.

Ante el ascenso de los esquemas de producción flexibles iniciado desde la década de los años sesenta, así como la consecuente crisis del fordismo en el primer lustro de los años setenta, la metropolización y la urbanización son nido del desarrollo terciario y aspectos importantes para los servicios en la economía nacional.

La crisis de 1971-1973 se profundizó hasta 1982, donde las drásticas caídas de 1984 y 1987 marcaron gravemente la recesión del viejo esquema modernista ante la violencia del surgimiento de la producción flexible. Crisis que todavía encuentra acomodos en 1994, donde los problemas nacionales fueron parte de un reacomodo mundial. Las crisis asiática, rusa, argentina y turca durante los años noventa mostraron que estos reacomodos industriales a escala mundial, subordinados a la globalización del crédito, son vulnerables a las debilidades de un sistema basado en la especulación apátrida y un abusivo manejo financiero de la economía mundial.

Los reacomodos industriales en la geografía internacional se dan gracias a la plataforma de los mercados bursátiles que permiten circular ágil, libre y eficazmente el capital financiero en su 
forma de inversión extranjera neutra, directa e indirecta, que permite la reestructuración de la economía internacional en la relocalización territorial de la industria en una escala mundial. En esta relocalización los servicios financieros y los seguros son la logística que respalda y garantiza dichos reacomodos industriales con cobertura de riesgo. Los mercados de futuros y los derivados financieros son bastiones de arribo del capitalismo central a las regiones de capitalismo periférico. Por ello, las áreas donde más se concentra la inversión extranjera son las ciudades con alto grado de urbanización y terciarización, como las zonas metropolitanas, no tanto por su fuerte vínculo con mercados foráneos, sino también por ser áreas donde existe una alta consolidación de la logística adecuada para el desarrollo sano, ágil y garantizado de la prosperidad de negocios transnacionales.

Dentro de las actividades manufactureras, la industria maquiladora encuentra su acomodo en el nuevo esquema, mientras que aquéllas de tipo más tradicional, que no son filiales transnacionales, sino de capitales propios y nacionales, de menor envergadura y monto, crecen de manera más lenta y tienen anhelos de desarrollo más local y modesto.

En esta fase de terciarización de la economía, la transformación de las metrópolis va hacia una gestión de servicios donde las industrias se deslocalizan mientras se concentran los servicios y las inversiones. En este proceso existe una migración de trabajadores de áreas donde su esfuerzo tiene una productividad marginal a aquéllas donde ésta es mayor, sobre todo en sectores más modernos y transnacionales.

La presencia de inversión extranjera directa en las zonas metropolitanas es muy importante, por ejemplo, en el 2006 más de $60 \%$ de este tipo de inversión se concentró en la Zona Metropolitana de la Ciudad de México (ZMCM), que aparece sombreada en el mapa I.

Dentro del desarrollo metropolitano de México, la ZMCM es de especial interés, pues es allí donde gravita todo el sistema de ciudades del país. Por ello, es importante comprender que dicho sistema tuvo cambios en sus patrones como consecuencia del surgimiento del esquema posfordista que afectaron la migración y la distribución territorial de la población de todo el país.

Este nuevo esquema, similar al modernista, es abiertamente antiagrario por sus fuertes montos migratorios del campo a la ciudad, incluso migración urbana-urbana, por lo que la PEA agrícola cada día es menor. Algunas tendencias en las transformaciones socioocupacionales más importantes son: 


\section{Mapa I \\ Localización de la Zona Metropolitana de la Ciudad de México}

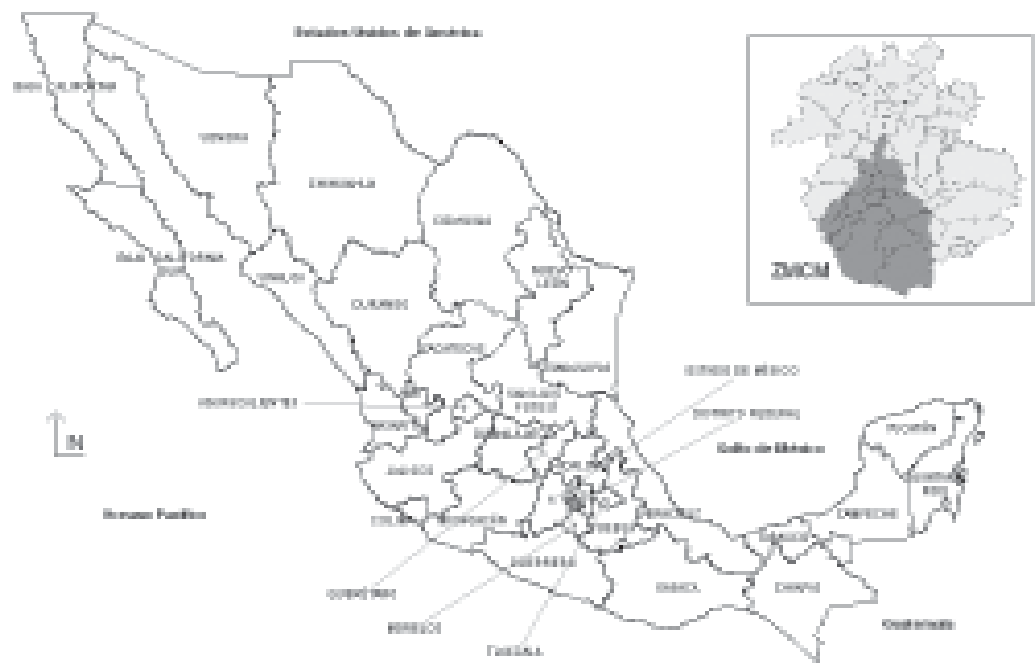

Fuente: Elaboración de Izunsa (2005) con base en Cartografía Digital, 1998, INEGI, México.

- La expansión y diferenciación de los estratos no manuales. Los trabajos técnicos operacionales manuales, como los artesanales, pierden presencia ante los trabajos de servicios de cuello blanco, como profesionistas y servidores públicos, bancarios, de seguros y algunos de tipo comercial.

- Aumento del sector popular urbano. Las áreas urbanas y metropolitanas cada vez concentran mayor población debido al alto grado de inmigración.

Los trabajos no manuales especializados en la producción crecen de manera significativa y se vinculan al desarrollo tecnológico de los procesos de producción y su vigencia, como nivel de capitalización, van de la mano con los negocios y organizaciones con que se vinculan. No obstante, los trabajos no manuales son los que encuentran mayor acomodo en la logística y administración de los procesos productivos propios de la producción flexible altamente automatizados. Al parejo, se dan también aquellos servicios en materia financiera y de cobertura de riesgo, como los seguros, asimismo algunos servicios públicos se acogen a la logística de la promoción en la comercialización y el financiamiento de los negocios. El comercio, por otra parte, también 
prospera pero crece siempre y cuando esté integrado, como nexo intermedio o dentro de la esfera de la producción, porque aquéllos especializados en el consumo final son sobre todo informales. En el medio rural domina la producción y explotación directa de los recursos naturales, pues, como se mencionó, la terciarización de la economía es un fenómeno urbano.

La transición del régimen de producción rígida al de producción flexible tiende a acrecentar el fenómeno de concentración de la fuerza laboral en las áreas urbanas y metropolitanas. En México esta concentración rompe con la tendencia propia del periodo endogenista, puesto que al ser una economía oligoproductora, la poca diversidad industrial genera escasas posibilidades de diversificar y explotar todas las potencialidades que el territorio otorga a la población, por lo que ésta se concentra en pocos polos de desarrollo.

En el nuevo régimen de producción flexible -no sólo por la apertura económica y las políticas de desconcentración industrial y descentralización de la vida política del país, sino por efecto de la presencia de una gran diversidad de negocios transnacionales localizados en el territorio nacional- las potencialidades territoriales se aprovechan mejor, lo que explica que las zonas metropolitanas del país, que tradicionalmente concentraban gran parte de la población, ahora pierdan vigor en su crecimiento, todavía alto pero en menor medida, y que las áreas del territorio que otorgan oportunidades locales y regionales a los inversionistas atraigan inmigrantes. Por ello, el régimen de producción flexible no resta fuerza a la concentración poblacional, pero en el caso de México, el esquema aporta nuevas vocaciones industriales oportunas para el desarrollo local. De esta manera, las ciudades medias y fronterizas así como los puertos comienzan a atraer población, mientras el campo se sigue despoblando cada vez de manera más acentuada porque sus habitantes ahora tienen muchos más destinos.

El mercado laboral también multiplica sus estructuras al tiempo que se distancian tanto los niveles de remuneración como de estatus social. Asimismo, la organización del territorio muestra una gran diversidad en cuanto a vocaciones industriales que, a su vez, expresa como polarización social la grave y cada vez más acentuada división del trabajo.

Las jerarquías urbanas también se vieron modificadas, por ejemplo, en el sistema de ciudades de los años noventa donde la primacía de la ZMCM encabezaba verticalmente todo el esquema. Ahora su importancia ha disminuido y otras zonas metropolita- 
nas, como la de Guadalajara, han ganado terreno. Siguen siendo subsistemas de la ZMCM pero con menor verticalidad pues estas nuevas zonas tienen una mayor autonomía regional y local, así como una interrelación más armoniosa y equidistante (equiparidad) más propia para un desarrollo económico que se funda en una mejor organización del territorio.

En el mapa II se identifica la división administrativa pública del Distrito Federal y de los municipios conurbados. Los municipios del Estado de México muestran una alta conurbación con el Distrito Federal, por lo que éste poco a poco respecto del área conurbada. En el cuadro 1 se observa, de 1950 a 2000, la participación de la población residente en el DF respecto de los municipios conurbados que conforman la ZMCM. En 1950 el DF albergaba $66 \%$ de la población que vivía en el área de la ciudad central; para el año 2000 sólo alberga a 9\%.

\section{Mapa II}

Delimitación de la ZMCM, 2000

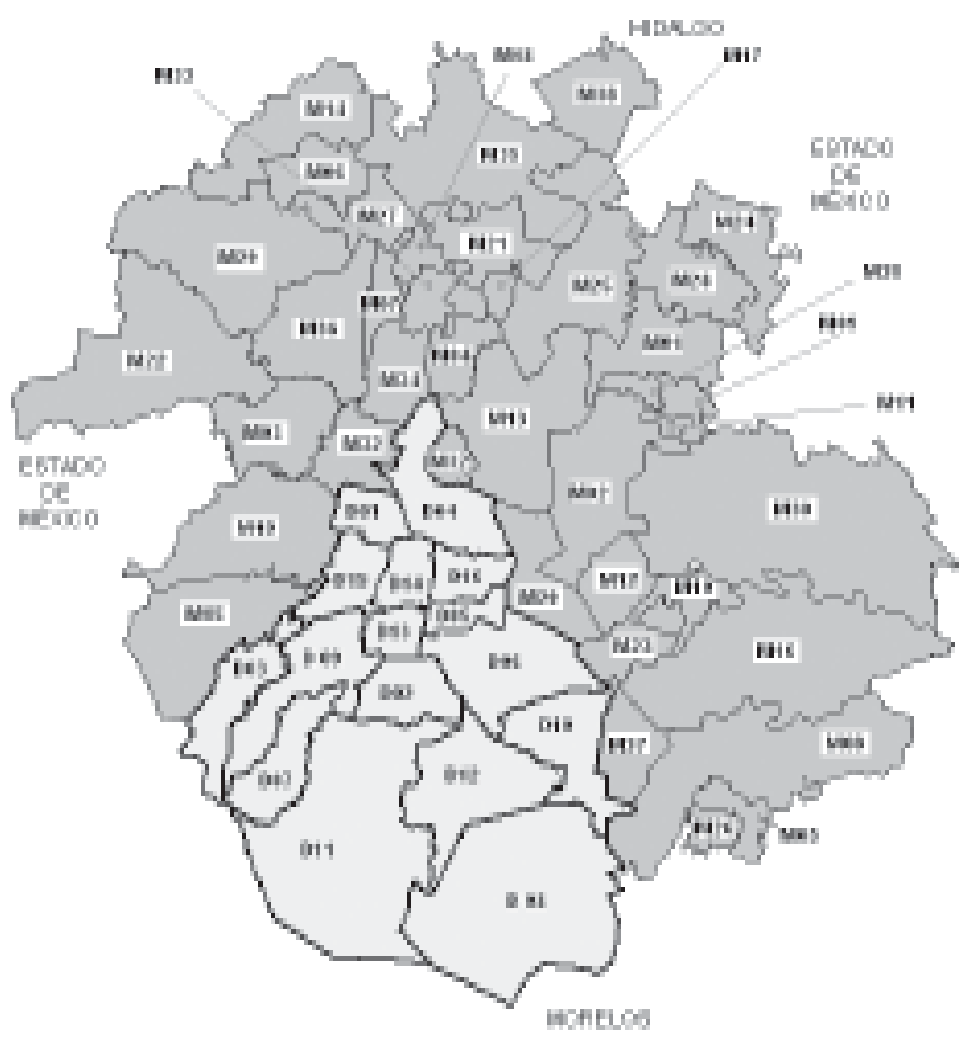


continuación...

\begin{tabular}{lll}
\hline \multicolumn{1}{c}{ Delegaciones } & \multicolumn{1}{c}{ DF } & \multicolumn{1}{c}{ Municipios } \\
\hline D01 Azcapotzalco & M01 Acolman & M20 Nezahualcóyotl \\
D02 Coyoacán & M02 Atenco & M21 Nextlalpan \\
D03 Cuajimalpa & M03 Atizapán & M22 Nicolás Romero \\
D04 Gustavo A. Madero & M04 Coacalco & M23 La Paz \\
D05 Iztacalco & M05 Cocotitlán & M24 San Martín de Las \\
& M06 Coyotepec & M25 Tecámac \\
D06 Iztapalapa & M07 Cuautitlán & M26 Temamatla \\
D07 Magdalena Contreras & M08 Chalco & M27 Teoloyucan \\
D08 Milpa Alta & M09 Chiautla & M28 Teotihuacán \\
D09 Álvaro Obregón & M10 Chicoloapan & M29 Tepotzotlán \\
D10 Tláhuac & M11 Chiconcuac & M30 Texcoco \\
D11 Tlalpan & M12 Chimalhuacán & M31 Tezoyuca \\
D12 Xochimilco & M13 Ecatepec & M32 Tlalnepantla \\
D13 Benito Juárez & M14 Huehuetoca & M33 Tultepec \\
D14 Cuauhtémoc & M15 Huixquilucan & M34 Tultitlán \\
D15 Miguel Hidalgo & M16 Ixtapaluca & M35 Zumpango \\
D16 Venustiano Carranza & M17 Jaltenco & M36 Cuautitlán Izcalli \\
& M18 Melchor Ocampo & M37 Valle De Chalco \\
& & Solidaridad \\
& M19 Naucalpan & M38 Tizayuca (Hidalgo) \\
\hline
\end{tabular}

Fuente: Elaboración de Izunsa (2005) con base en: Conapo, La población en México: situación actual y desafíos futuros, 2000 y Cartografía Digital, INEGI, 1996.

\section{Cuadro 1}

ZMCM. Participación porcentual de la población, 1950-2000

\begin{tabular}{lrrrrrr}
\hline \multicolumn{1}{c}{$\%$} & 1950 & 1960 & 1970 & 1980 & 1990 & 2000 \\
\hline Distrito Federal & 90 & 88 & 76 & 62 & 54 & 48 \\
Ciudad Central* & 66 & 51 & 32 & 18 & 13 & 9 \\
Municipios conurbados & 10 & 12 & 24 & 38 & 46 & 52 \\
ZMCM & 100 & 100 & 100 & 100 & 100 & 100 \\
\hline
\end{tabular}

* Comprende las delegaciones Cuauhtémoc, Benito Juárez, Miguel Hidalgo y Venustiano Carranza.

Fuente: Elaboración de Izunsa (2005) con base en: INEGI, Censo General de Población y Vivienda, 1950, 1960, 1970 y 2000 para el D.F., Estado de México y estado de Hidalgo, México.

La expansión de la mancha urbana y la migración de población del DF a los municipios conurbados del Estado de México expresan que para 1950 la población del DF representaba 90\%, el 10\% restante vivía en el área conurbada del Estado de México. Pero para el año 2000 la población del DF es apenas 48\% del total residente en el área que comprende la zMCM.

Esta disminución se debe a dos razones principales: al crecimiento de la población residente en la ZMCM, sobre todo por efec- 
to de la inmigración y por la terciarización de la economía dentro del área del DF que orienta el uso del suelo urbano a espacios de comercio, servicios financieros y seguros, así como de servicios públicos. Las zonas habitacionales se sustituyen paulatinamente por este otro tipo de uso del suelo y en consecuencia la población es desalojada o bien abandona el área y la enajena a los negocios terciarios que dan fuerte plusvalía a estos terrenos.

En el mapa III se muestra la transición demográfica de la ciudad de México, como lo analiza Izunsa (2005), por efecto de los dos causales anteriormente señalados.

\section{Mapa III}

\section{ZMCM. Etapas de transición demográfica, 2000}

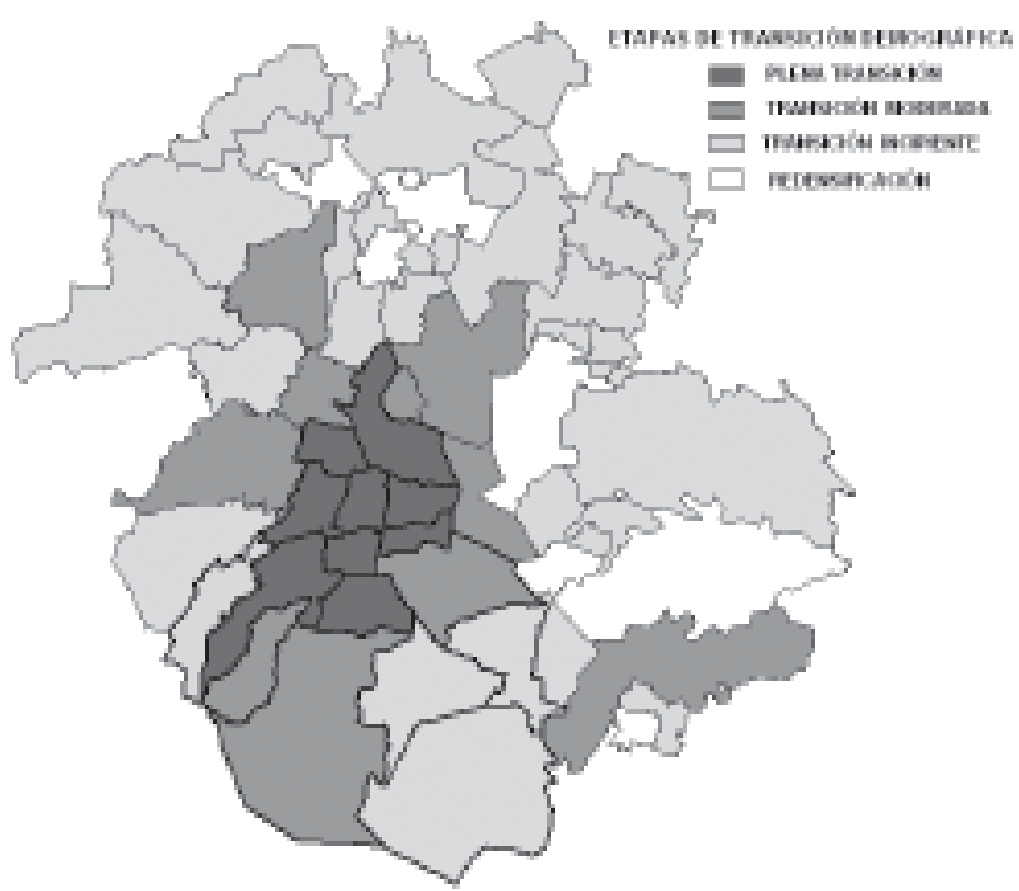

Fuente: Elaboración de Izunsa (2005) con base en Censo General de Población y Vivienda, 1950-2000, INEGI.

Esta transición se explica principalmente tanto por el efecto de la inmigración de la provincia por la ZMCM, cómo por los reacomodos dentro del área de la población originalmente residente debido al cambio de patrones en la dinámica de metropolización ante el nuevo esquema de producción flexible, la terciarización de las economías urbanas y el nuevo uso del suelo urbano. 
En el mapa IV se ven las etapas de expansión urbana de la ZMCM, lo que muestra cómo se ha dado esta ocupación del territorio y del crecimiento de la ciudad de México desde el DF como zona central o de origen hacia las periferias, lo que indica claramente el desarrollo periurbano de la ciudad de México de 1950 a 2000.

\section{Mapa IV}

ZMCM. Etapas de expansión urbana

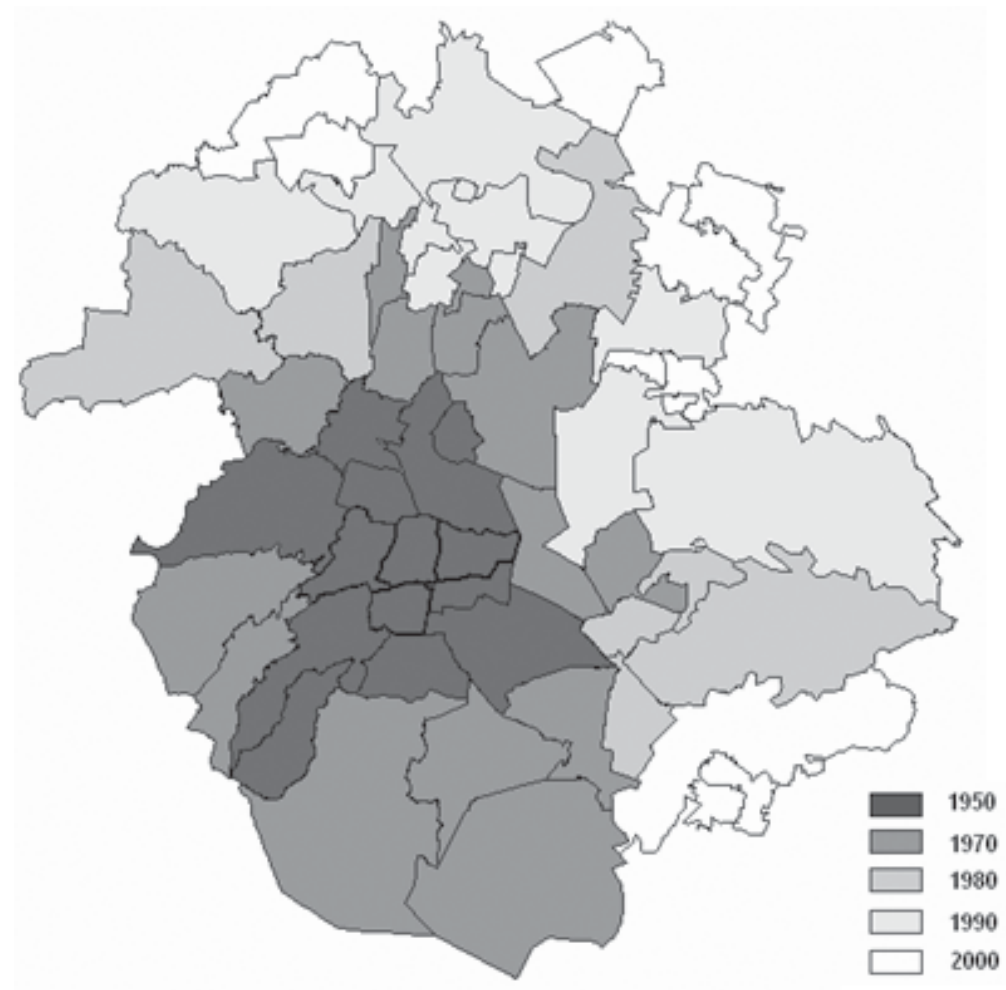

Fuente: Elaboración de Izunsa (2005) con base en Censo General de Población y Vivienda, 1950-2000, INEGI.

El trabajo de Izunsa es bastante profundo en el análisis de la expansión urbana y la nueva dinámica metropolitana de la ciudad de México. Sin embargo, no aborda el tema de interés para esta investigación que radica principalmente en ver cómo se comporta la sociología del trabajo ante el surgimiento de los mercados urbanos, ante la producción flexible y, sobre todo, los efectos en el bienestar de la población.

Las zonas metropolitanas del país se integran a regiones distintas, por ello el análisis regional es imprescindible para la com- 
prensión del presente estudio. Ante el nuevo esquema posfordista, las regiones no entran en conflicto, pero sí se vuelven más autónomas. Las diferencias ocupacionales son distintas de una población a otra, entre localidades y de región a región. Su especialización en el mapa nacional de desarrollo causa una interdependencia entre las mismas como parte de un esquema integrador y consistente.

No obstante, y deseando que este postulado fuera del todo cierto, debe admitirse que existen zonas de atraso y abierta marginación en esta geografía económica. Lo que es más, la mayor parte de estas zonas observadas en 1990, quince años después revelan que la marginación ha aumentado.

El desarrollo nacional como parte del régimen de producción flexible, y como área del capitalismo periférico, no ha sido el mismo para todas las regiones del país. Mientras vemos un sistema urbano en ascenso, también existen grandes áreas de abierta y creciente marginación (véase cuadros 5 y 6 y mapas V-IX).

Respecto a la terciarización de la economía, en las áreas metropolitanas se concentra la mano de obra pero en una dicotomía cada vez más contradictoria. Por ejemplo, en los servicios a la producción está la mano de obra más calificada con los mejores niveles salariales y de estatus social, mientras que en los servicios al consumo hay una abierta precarización, un lumpenproletariado que domina las actividades informales.

A su vez, las labores manuales en el sector secundario están peor remuneradas y cada vez tienen menor número de trabajadores respecto de los operarios y técnicos profesionales, quienes mejoran su nivel de estatus e ingreso per cápita. Con todo, el sector secundario expulsa mano de obra al sector terciario, especialmente de los servicios al consumo: comercio informal.

Los servicios al productor se componen de actividades de tipo bancario, financiero, seguros, mercadotecnia, comercialización, consultorías y otros servicios profesionales. En los servicios al consumidor se tienen actividades de trabajo doméstico, vendedores ambulantes, talleres familiares, labores manuales temporales, entre otras.

Los servicios al consumidor se especializan en la esfera de los bienes finales, mientras que los servicios al productor atienden aspectos relativos a los bienes intermedios al productor. Por ello, la geografía de la localización industrial determina la propia geografía de la especialización de los servicios tanto al productor como al consumidor. Así, los servicios de un lugar específico crean su propia conformación social y laboral, dando con ello la divi- 
sión y especialización de los servicios en un ámbito geográfico. De esta manera se tienen patrones propios de localización regional y local de cierto tipo de servicios desde el lado de la oferta, que son a su vez las razones de las asimetrías en el desarrollo regional y local.

La terciarización de las grandes ciudades es un proceso evidente en la fase posfordista. Las áreas metropolitanas reciben un creciente flujo de mano de obra que se ocupa en actividades terciarias. No obstante, la dicotomía entre un sector moderno de la economía y uno tradicional expresa otra dicotomía que necesariamente es concomitante al proceso de terciarización, esto es, la división entre los servicios a la oferta y la de los servicios al consumo. Entre los servicios a la producción también se observa otra dicotomía de interés: entre los directivos con cargos a largo plazo y los mandos medios y personal administrativo con trabajos temporales. Las desigualdades vistas en el proceso de terciarización son efecto de cómo las industrias organizan su producción, por lo que en el fondo del problema debe quedar clara la lógica que imprimen las nuevas relaciones industriales en la producción y en la circulación y, con ello, en los servicios y el mercado laboral. La terciarización de la economía se explica por distintos factores. Primero, el aumento en la composición orgánica del capital, lo que provoca que en las fábricas las máquinas sustituyan a un buen número de trabajadores que deben colocarse en otras ramas de actividad económica. Segundo, las crisis económicas que obligan a los trabajadores desempleados a colocarse en el sector informal. Tercero, el nuevo régimen de producción flexible que inaugura una nueva forma de relación industrial basado en la flexibilidad laboral, las empresas outsourcing y el nuevo sindicalismo democrático.

En la terciarización es interesante observar la polarización entre los servicios a la producción como a la comercialización.

Mientras que las actividades primarias son pocas, las secundarias son múltiples y las terciarias tienden a una enorme gama de actividades muy distintas. Por ello el sector terciario presenta problemas para un estudio acucioso, pues su enorme diversidad dificultad su tipificación e identificación ante otras que muchas veces parecen iguales o similares, pero al estudiarlas más de cer$\mathrm{ca}$, se ve que los servicios tienen aspectos muy singulares.

La clasificación de los servicios en un nivel sectorial incluye actividades muy diversas que hacen más complejo y complicado su estudio. En los procesos manufactureros, por ejemplo, aparecen una enorme cantidad de servicios que atiendan competitiva- 
mente algunos de los aspectos de la producción. Asimismo, los sectores manufactureros propios del esquema de producción flexible tienen servicios muy diversos y complejos respecto de sus fases de factorización.

Cabe destacar que en los medios rural, urbano y metropolitano el desempleo es un fenómeno persistente. Los servicios al productor y al consumidor se concentran especialmente en el medio urbano, sobre todo en las grandes metrópolis y están dirigidos a actividades financieras y de la banca, aseguradoras, asesoría, profesionales en la adquisición de insumos y distribución de mercadería -como compra venta de equipo-, herramientas e insumos industriales.

Los servicios colectivos son los que están destinados al bienestar de la comunidad, son los menos representativos y parten de la iniciativa privada para ofrecer a la población educación, investigación, seguridad social. Aunque por ahora es el sector que crea menos empleos, aumenta de manera rápida en las áreas urbanas y metropolitanas. Esto es parte de la concesión a la iniciativa privada de los servicios que el Estado tradicionalmente brinda a la población.

La producción flexible ha implicado un cambio en el papel económico del Estado, como una nueva visión de éste como objeto económico. Para la última mitad del siglo xx, Brenner concibe el cambio del papel del Estado ante la reestructuración posfordista y sus formas de distribución espacial. La figura I fue tomada de este autor, quien dice que "Hacia fines del siglo Xx, por tanto, la propia organización espacial del Estado se ha convertido en una importante arma locacional en la competencia interespacial entre ciudades, regiones y estados en la economía mundial" (Brenner, 2003).

En este sentido, la relocalización industrial y la desconcentración del aparato productivo nacional, así como las políticas de descentralización y el nuevo federalismo, implican que progrese este tipo de servicios comunitarios por parte de la iniciativa privada.

Con el aumento del desempleo urbano por la expulsión de mano de obra de los sectores primario y secundario, el sector terciario observa un incremento, en especial, en el comercio ambulante con carácter informal, baja capitalización y ganancias exiguas que son el sustento del trabajador.

Se observa que paulatinamente el mercado interno pasa a un segundo plano y aumentan las ventas hacia el mercado externo, sobre todo por el acelerado crecimiento de la industria maquiladora de exportación que se localiza en las áreas periféricas de la 


\section{Figura I}

Formas de organización espacial del Estado

Urbanización, formas de Estado y la economia mundial, 1950-2000

FORMAS DE ORGANIZACION ESPACIAL DEL ESTADO

\begin{tabular}{|l|c|c|}
\hline & $\begin{array}{c}\text { ESTADO } \\
\text { TERRITORIAL } \\
\text { NACIONAL }\end{array}$ & $\begin{array}{c}\text { ESTADO } \\
\text { TERRITORIAL } \\
\text { "GLOCAL" }\end{array}$ \\
CIUDAD COMO & $\begin{array}{c}\text { Desarrollismo nacional: las } \\
\text { COORDENADA } \\
\text { DE PODER ESTATAL } \\
\text { de transmisión de la politica } \\
\text { económica nacional; surgi- } \\
\text { miento de la ciudad "gerencial" } \\
\text { keynesiana }\end{array}$ & $\begin{array}{c}\text { "Glocalización" del poder del } \\
\text { Estado: la política de las } \\
\text { ciudades es reorientada hacia } \\
\text { la promoción de estrategias de } \\
\text { crecimiento endógeno; } \\
\text { proliferación de iniciativas } \\
\text { económicas locales; el poder } \\
\text { del Estado es re-escalado } \\
\text { hacia abajo hacia instituciones } \\
\text { locales y regionales }\end{array}$ \\
\hline
\end{tabular}

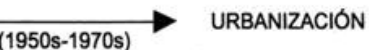

(post-1970s)

\begin{tabular}{|c|c|c|}
\hline $\begin{array}{l}\text { MARGEN } \\
\text { COMO NODO } \\
\text { DE ACUMULACION }\end{array}$ & $\begin{array}{l}\text { La ciudad sirve como motor de } \\
\text { crecimiento económico nacio- } \\
\text { nal; predominio de la ciudad } \\
\text { industrial fordista; la ciudad } \\
\text { sirve como "máquina de } \\
\text { crecimiento" y como lugar de } \\
\text { consumo colectivo e inversiones } \\
\text { estatales en bienes públicos }\end{array}$ & $\begin{array}{l}\text { Formación de la ciudad global: } \\
\text { desacoplamiento del crecimien- } \\
\text { to urbano del crecimiento de las } \\
\text { economías nacionales; intensi- } \\
\text { ficación de la competencia } \\
\text { inter-espacial entre ciudades y } \\
\text { regiones a escala mundial; } \\
\text { explosión de desarrollo } \\
\text { especial desigual a todas las } \\
\text { escalas }\end{array}$ \\
\hline & $\begin{array}{c}\text { ECONOMIA } \\
\text { INTERNACIONAL }\end{array}$ & $\longrightarrow \quad \begin{array}{c}\text { ECONOMIA } \\
\text { GLOBAL }\end{array}$ \\
\hline
\end{tabular}

ORGANIZACIÓN ESPACIAL DE LA ECONOMIA MUNDIAL

Zona Metropolitana de la Ciudad de Gudalajara (ZMCG), así como el incremento de los sectores comercial y de servicios. Afirmación con la que coincide Arroyo (1993).

Por otra parte, la PEA, algo más de la tercera parte en la ZMCG, se ocupa en el sector secundario, proporción que viene a ser cada vez menor pues en 1970 la PEA industrial rondaba 40\% y para el 2006 se calcula en la tercera parte. Al mismo tiempo, el sector terciario aumenta debido a que capta esa PEA industrial además de los desempleados rurales. Para el 2006 se estima que ésta ya es 60\% de la PEA, siendo que en 1970 era de 47\%. Aproximadamente la mitad del crecimiento del sector terciario proviene del sector secundario y la otra mitad del primario. 
Hay una tendencia a la desconcentración espacial de las actividades industriales ya que muchas de ellas se trasladan a áreas periféricas y satelitales a la ZMCG. Por su parte, la inversión extranjera directa se coloca en áreas cercanas a la ciudad pero en parques y distritos industriales. Lo que en el periodo 1950-1990 no era evidente, desde la última década de la centuria ya es una realidad: la desconcentración industrial de la ZMCG hacia sus áreas periféricas y satelitales. Aunque hay que decir que esta afirmación puede considerarse una hipótesis secundaria que articula todo el trabajo pero no sirve de hilo conductor, ya que aún existe controversia al respecto.

La ZMCG sigue creciendo, aunque a un ritmo menor que de 1950 a 1980, con todavía un alto nivel de atracción poblacional. Los problemas concomitantes de este crecimiento son: el caos en el desarrollo urbano y en el ordenamiento del suelo, deterioro del medio ambiente y problemas en la calidad y dotación de servicios públicos e infraestructura.

La industria jalicience presenta una de las más altas concentraciones del país en una sola región metropolitana integrada por los municipios de Guadalajara, Zapopan, Tlaquepaque, Tonalá, Zapotlanejo, Acatlán de Juárez, Tlajomulco de Zúñiga, El Salto y Chapala (estas últimas tres incorporadas recientemente). En esta área se albergan tres cuartas partes de la industria localizada en el estado de Jalisco y más de $90 \%$ de la población ocupada. Después de la ZMCM, es la zona industrial con una concentración más alta; pero en términos de distribución territorial es la más grande del país.

Este fenómeno de desconcentración industrial de las zonas metropolitanas no es particular de Guadalajara, sino de todas las áreas metropolitanas del país: la ZMCM, la de Monterrey, la de Puebla, así como fenómenos de conurbación metropolitana en ciudades del corredor industrial del Bajío.

\section{Marginación, migración y pobreza urbana}

Un análisis comparativo entre la geografía de la marginación en México en los años de 1995 y $2000^{1}$ muestra que, primero, la marginación en general no ha disminuido en grado sino que se ha recompuesto. La marginación, según estudios del Conapo, se mide con los siguientes parámetros: muy baja, baja, media, alta y muy

${ }^{1}$ Estudios del Consejo Nacional de Población (Conapo) sobre la marginación en México para 1995 y 2000. 
alta. Segundo, la pobreza aumentó en las zonas tradicionalmente marginales y son muy pocas las áreas que observan una disminución de la misma. Tercero, el fenómeno de la marginación no es estable, debido al crecimiento natural de la población y a la migración, lo que explica su recomposición y redistribución en el territorio.

La recomposición de la pobreza se da porque en algunas localidades aumenta o disminuye debido a que la población no mantiene una residencia estable. Así, es difícil que las políticas públicas dirigidas a abatir la pobreza tengan éxito. Un estudio en el ámbito municipal puede arrojar algunos rasgos del fenómeno tanto a nivel local como regional. En el caso del estado de Guanajuato, durante el periodo estudiado el índice de marginación para el cinturón industrial se mantiene bajo, pero en el noroeste de la entidad aumenta de media a alta, así como una parte del sureste y otra del noreste. Lo que demuestra que, en términos generales, la marginación aumentó en este estado.

En cuanto a lo nacional existe una tendencia migratoria de las áreas marginales del sur y centro del país hacia la frontera norte. Esto genera un fenómeno de contagio, pues esta región, especialmente Sonora, Chihuahua y Coahuila, observa un aumento de la marginación: en 1995 era muy baja para casi todo su territorio y en el 2000 aparecen amplias extensiones con marginación también baja.

Esto abre una nueva línea de investigación en torno al problema que se plantea si la marginación aumenta, disminuye o bien se reacomoda y reexpresa una geografía dinámica donde ocurre un crecimiento demográfico, especialmente por cuestiones sociales, que hace a pensar en contagios de una región a otra. Por ejemplo, en algunas áreas del sur y centro del país, al parecer, la marginación disminuye un poco de muy alta a alta, mientras que asimétricamente en el norte aumenta de muy baja a baja. Siendo el norte de la República una parte del territorio nacional donde se localizan fuertes montos de inversión extranjera, en especial en la creación de industrias maquiladoras, y donde la inversión pública federal es alta, como en las carteras de crédito bancario, en términos de densidad poblacional la marginación es de las más bajas del país. Su aumento se debe a la fuerte densidad poblacional resultado de la inmigración del sur y centro de la nación. Lo anterior puede indicar un contagio de las zonas de alta marginación del resto de la República a esta franja.

En un caso más local, la ciudad fronteriza de Tijuana crece a tasas que promedia entre 11 y 13 puntos porcentuales al año. Es 


\section{Grado de marginación por entidad federativa 1990, 2000 y 2005}

\begin{tabular}{|c|c|c|c|c|c|}
\hline $\begin{array}{c}\text { Entidad } \\
\text { Federativa }\end{array}$ & 1990 & $\begin{array}{l}\text { Grado } \\
2000\end{array}$ & 2005 & $\begin{array}{c}\text { Situación } \\
\text { Cambio en } \\
\text { el grado de } \\
\text { marginación }\end{array}$ & $\begin{array}{c}\text { Se califica } \\
\text { como: }\end{array}$ \\
\hline Distrito Federal & Muy bajo & Muy bajo & Muy bajo & Sin cambio & Persistente \\
\hline Baja California & Muy bajo & Muy bajo & Muy bajo & Sin cambio & Persistente \\
\hline Coahuila & Bajo & Muy bajo & Muy bajo & $\begin{array}{l}\text { Cambio } \\
\text { favorable }\end{array}$ & Progresivo \\
\hline Nuevo León & Muy bajo & Muy bajo & Muy bajo & Sin cambio & Persistente \\
\hline Chiapas & Muy alto & Muy alto & Muy alto & Sin cambio & Persistente \\
\hline Guerrero & Muy alto & Muy alto & Muy alto & Sin cambio & Persistente \\
\hline Oaxaca & Muy alto & Muy alto & Muy alto & Sin cambio & Persistente \\
\hline Durango & Alto & Medio & Medio & $\begin{array}{l}\text { Cambio } \\
\text { favorable }\end{array}$ & Progresivo \\
\hline Guanajuato & Alto & Alto & Medio & $\begin{array}{l}\text { Cambio } \\
\text { favorable }\end{array}$ & Progresivo \\
\hline Nayarit & Medio & Alto & Medio & $\begin{array}{l}\text { Cambio } \\
\text { favorable }\end{array}$ & $\begin{array}{l}\text { Reincidente } \\
\text { progresivo }\end{array}$ \\
\hline Querétaro & Alto & Medio & Medio & $\begin{array}{l}\text { Cambio } \\
\text { favorable }\end{array}$ & Progresivo \\
\hline Sinaloa & Medio & Medio & Medio & Sin cambio & Persistente \\
\hline Tlaxcala & Medio & Medio & Medio & Sin cambio & Persistente \\
\hline Zacatecas & Alto & Alto & Medio & $\begin{array}{l}\text { Cambio } \\
\text { favorable }\end{array}$ & Progresivo \\
\hline Aguascalientes & Bajo & Bajo & Bajo & Sin cambio & Persistente \\
\hline Baja California Sur & Bajo & Bajo & Bajo & Sin cambio & Persistente \\
\hline Chihuahua & Bajo & Bajo & Bajo & Sin cambio & Persistente \\
\hline Colima & Bajo & Bajo & Bajo & Sin cambio & Persistente \\
\hline Jalisco & Bajo & Bajo & Bajo & Sin cambio & Persistente \\
\hline México & Bajo & Bajo & Bajo & Sin cambio & Persistente \\
\hline Morelos & Bajo & Medio & Bajo & $\begin{array}{l}\text { Cambio } \\
\text { favorable }\end{array}$ & $\begin{array}{l}\text { Reincidente } \\
\text { regresivo }\end{array}$ \\
\hline Quintana Roo & Medio & Medio & Bajo & $\begin{array}{l}\text { Cambio } \\
\text { favorable }\end{array}$ & Regresivo \\
\hline Sonora & Bajo & Bajo & Bajo & Sin cambio & Persistente \\
\hline Tamaulipas & Bajo & Bajo & Bajo & Sin cambio & Persistente \\
\hline Campeche & Alto & Alto & Alto & Sin cambio & Persistente \\
\hline Hidalgo & Muy alto & Muy alto & Alto & $\begin{array}{l}\text { Cambio } \\
\text { favorable }\end{array}$ & Regresivo \\
\hline Michoacán & Alto & Alto & Alto & Sin cambio & Persistente \\
\hline Puebla & Muy alto & Alto & Alto & $\begin{array}{l}\text { Cambio } \\
\text { favorable }\end{array}$ & Progresivo \\
\hline San Luis Potosí & Alto & Alto & Alto & Sin cambio & Persistente \\
\hline Tabasco & Alto & Alto & Alto & Sin cambio & Persistente \\
\hline Veracruz & Muy alto & Muy alto & Alto & $\begin{array}{l}\text { Cambio } \\
\text { favorable }\end{array}$ & Progresivo \\
\hline Yucatán & Alto & Alto & Alto & Sin cambio & Persistente \\
\hline
\end{tabular}

Fuente: Consejo Nacional de Población 1990, 2000 estimaciones para el 2005 de CONEVAl con base en el il Conteo de Población y Vivienda. Ordenado por el autor. 
la localidad con mayor crecimiento demográfico del país. Asimismo, las áreas marginales de extrema pobreza son, de la mancha urbana, las más extensas, y aumentan a gran velocidad.

En el cuadro 6 se presentan doce entidades con sus distintos niveles de marginación en la República. Chiapas, Guerrero, Oaxaca y Veracruz tienen los índices más altos. Estas entidades también son las de mayor intensidad migratoria. Chiapas muy bajo grado; Guerrero muy alto; Oaxaca medio y Veracruz bajo.

\section{Cuadro 6 \\ Índice de marginación}

\begin{tabular}{lccll}
\hline \multicolumn{1}{c}{ Entidad } & $\begin{array}{c}\text { Índice de } \\
\text { marginación }\end{array}$ & Lugar & $\begin{array}{c}\text { Gradode } \\
\text { marginación }\end{array}$ & $\begin{array}{c}\text { Gradode } \\
\text { intensidad } \\
\text { migratoria }\end{array}$ \\
\hline Michoacán & -0.44913 & 10 & Alto & Muy alto \\
Jalisco & -0.76076 & 25 & Bajo & Alto \\
Guanajuato & -0.07966 & 13 & Alto & Muy alto \\
Estado de México & -0.69469 & 21 & Bajo & Bajo \\
Distrito Federal & -1.52944 & 32 & Muy bajo & Muy bajo \\
Puebla & -0.72048 & 07 & Alto & Mediano \\
Veracruz & 1.27756 & 04 & Muy alto & Bajo \\
Guerrero & 2.11781 & 02 & Muy alto & Alto \\
Oaxaca & 2.07869 & 03 & Muy alto & Mediano \\
Hidalgo & -0.87701 & 05 & Muy alto & Alto \\
Chiapas & 2.25073 & 01 & Muy alto & Muy bajo \\
Zacatecas & -0.29813 & 12 & Alto & Muy alto \\
\hline
\end{tabular}

Fuente: Desarrollado por Berumen (2004). Información tomada de la Secretaría de Planeación del Desarrollo y Gasto Público. Gobierno del Estado de Sonora. En Miguel E. Berumen Barbosa, "La migración, puntal de la economía mexicana, http:// www.eumed.net/cursecon/ecolat/mx/mebb-migra.htm

Si se hace la misma correlación para las entidades con menor nivel de marginación se tiene lo siguiente: el Distrito Federal presenta la más baja marginación y su nivel migratorio también es muy bajo; Jalisco tiene bajo grado de marginación, pero alta intensidad migratoria; el Estado de México con bajo nivel de marginación presenta muy baja intensidad migratoria. Esto indica que la relación entre marginación y migración no es muy directa, más bien depende del grado de urbanización y de las ramas de actividad económica y de sus nexos con el nuevo esquema de producción flexible, o bien, cuando están al margen de este tipo de economías modernas, observan patrones propios de marginación y migración. 
Como se dijo, la migración y la marginación no tienen mucha relación, más bien son las vocaciones industriales locales y sus nexos con el desarrollo urbano e industrial, posmoderno o tradicional, las que explican los nuevos patrones de migración y distribución espacial de la población. Por otra parte, debe señalarse que esto expresa singularidades regionales en el país y que, por ejemplo, el desarrollo del occidente de México tiene patrones propios que no necesariamente comparte con otras regiones, lo que sucede con cada una de las que integran el territorio nacional.

Por otra parte, el efecto de las políticas públicas en el bienestar de la población, como revela el estudio de Maldonado y Palma (2006), tienen poco o nulo efecto. Las personas resuelven sus carencias, en especial las más vulnerables, normalmente mediante la emigración. En la nación hay una silenciosa movilización de miserables ${ }^{2}$ de las zonas más marginales a las menos, por lo que, según se planteó líneas atrás, es muy posible que los aumentos y disminuciones de marginación observados en la geografía se expliquen más por los reacomodos geográficos de los humildes, procurando nuevas y mejores oportunidades y una mayor calidad de vida, que por efecto de las políticas públicas a favor de disminuir la marginación.

Es posible que si un área disminuye su grado de marginación se deba a que los marginales se han ido; así como si un área aumenta su grado de marginación, sea el resultado de que existe inmigración de humildes al área. Las zonas de menor marginación atraen población, especialmente de las zonas más marginadas, expulsoras de población. Entonces, como en un sistema de vasos comunicantes, existe una fuerza que hace que la marginación se redistribuya en el territorio y tienda a uniformarse o bien a disminuir los contrastes y a atemperarse.

\section{Conclusión}

Es importante resaltar cómo la apertura económica en México afecta no sólo los reacomodos industriales en una nueva geografía, sino también la estructura de los mercados de trabajo.

La marginación en México no se resuelve, se reacomoda y reexpresa en una nueva geografía de la marginación y la pobreza donde, al parecer, las zonas que antes tenían muy alta marginación tienden a moverse a las de muy baja, de modo que en un

\footnotetext{
${ }^{2}$ El término no es peyorativo, se deriva de la palabra miseria, que es el efecto que se desea dar al hablar de marginación.
} 
segundo momento las zonas de muy alta ahora son sólo de alta, y las zonas de muy baja, ahora son de baja marginación. Este reacomodo de la pobreza evidencia que la calidad de vida y el bienestar de las personas se resuelve en lo inmediato de manera personal y no por los programas públicos para combatir la pobreza.

La precarización del campo continúa y en tiempos de posmodernidad este fenómeno se ha acrecentado y ahora comparte relevancia con el aumento del desempleo urbano y metropolitano que nutre al sector terciario, en especial en servicios al comercio, pero en actividades informales y ambulantes. El nuevo régimen de producción flexible es un abierto atentado laboral contra los campesinos cuyas masas engrosan los flujos de la migración internacional indocumentada, especialmente a la Unión Americana.

Se observa una abierta dicotomía en el sector terciario de la economía entre los servicios a la producción y los servicios al consumo. Los primeros con alto estatus tanto laboral como social, buenos salarios, así como empleos con larga duración, mismos que conviven con trabajadores dedicados a los servicios al consumo, de baja calificación y con trabajos temporales, mala remuneración y bajo estatus social, flexibles y con amplio desempleo friccional.

Un caso típico de este fenómeno es el de la ZMCG, donde se da una abierta desconcentración industrial a zonas periféricas y satelitales de la zona metropolitana, como también una tendencia creciente a la terciarización económica. La zona sigue atrayendo población, pero a menor ritmo que en décadas pasadas, y prosperan las actividades terciarias con la dicotomía antes mencionada. La ZMCG aún tiene un significativo grupo de micro, pequeñas y medianas empresas que actualmente acogen la mayor parte de la PEA, sin embargo, las grandes empresas ofrecen más nuevos empleos. Esto los servicios al productor, pero por parte de los servicios al consumidor, los empleos que más crecen son los informales, donde existe otra dicotomía entre los trabajos en servicios bancarios y financieros, de aseguradoras, de asesorías y de comercialización. Mientras que en el polo opuesto, el ambulantaje crece al parejo de los trabajadores mil usos donde caen los oficios. 


\section{Mapa V}

México: localidades con grado de marginación muy alto

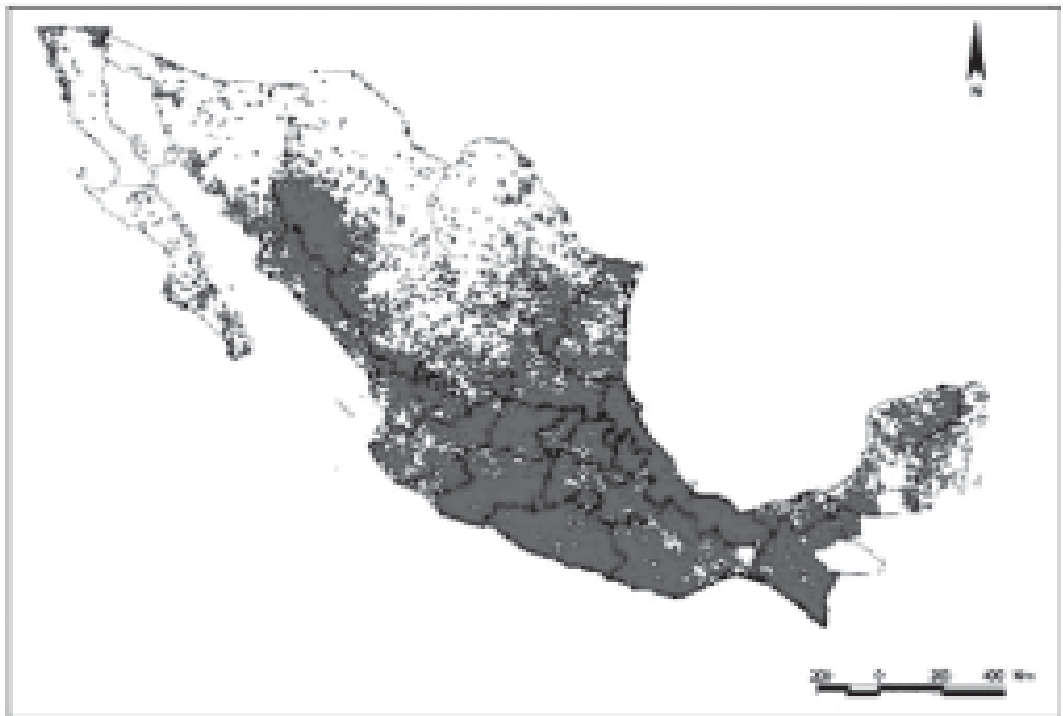

Fuente: Consejo Nacional de Población.

\section{Mapa VI \\ México: localidades con grado de marginación alto}

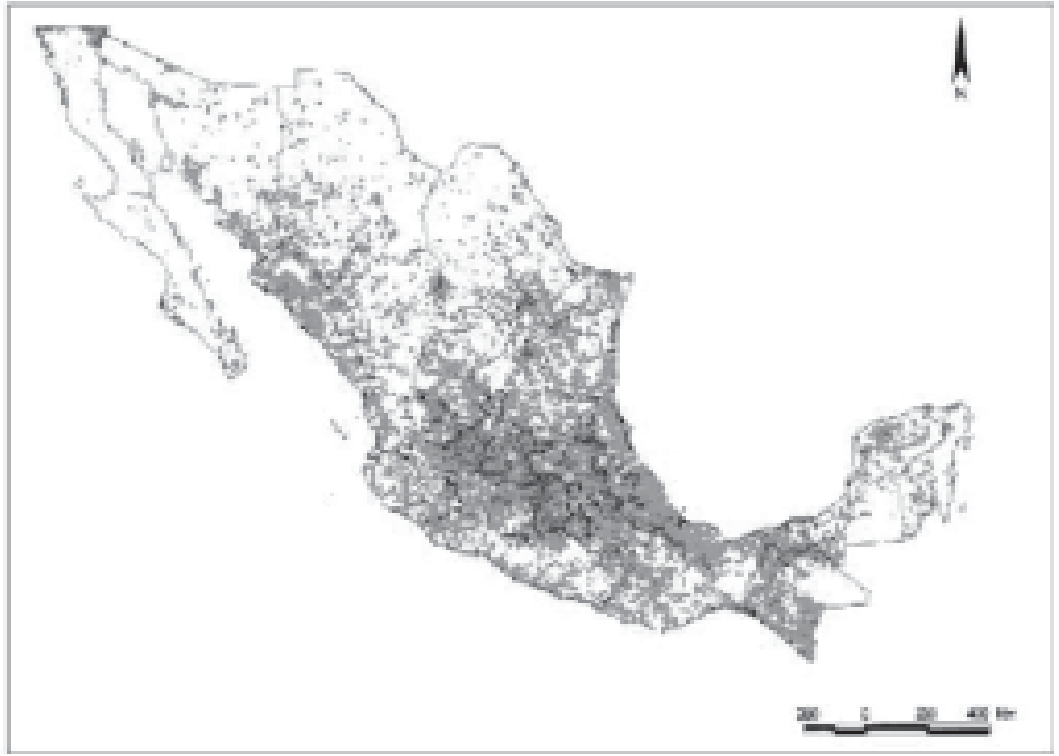

Fuente: Consejo Nacional de Población. 


\section{Mapa VII}

México: localidades con grado de marginación medio

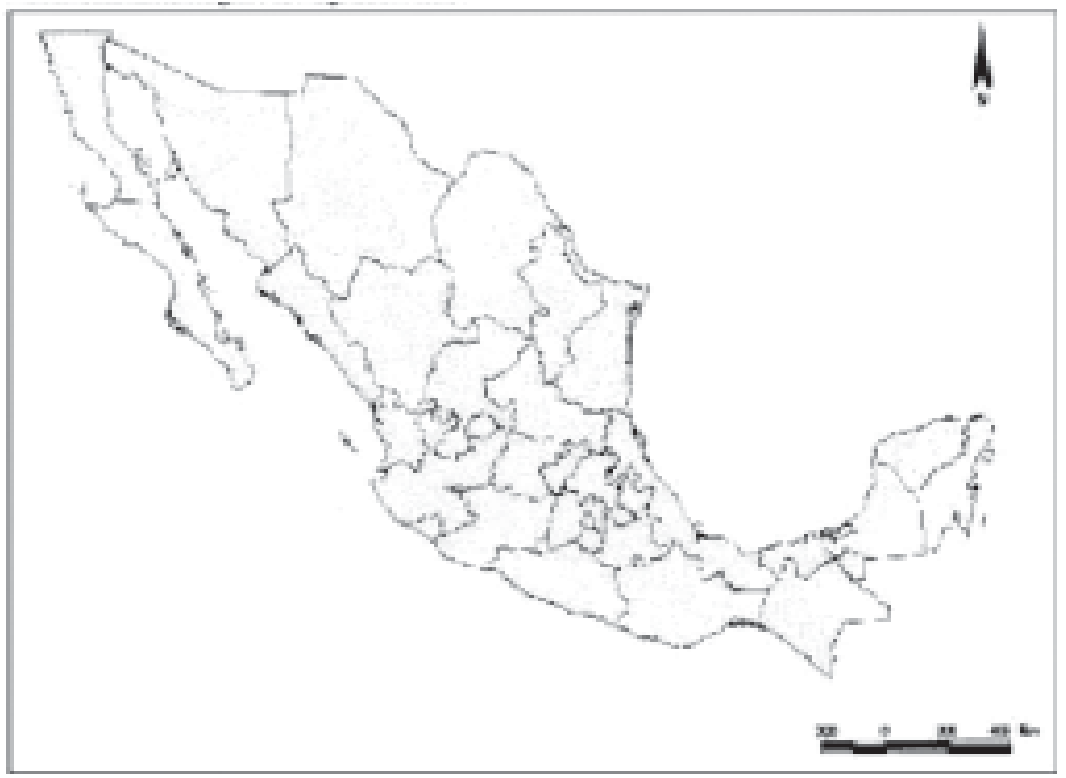

Fuente: Consejo Nacional de Población.

\section{Mapa VIII}

México: localidades con grado de marginación bajo

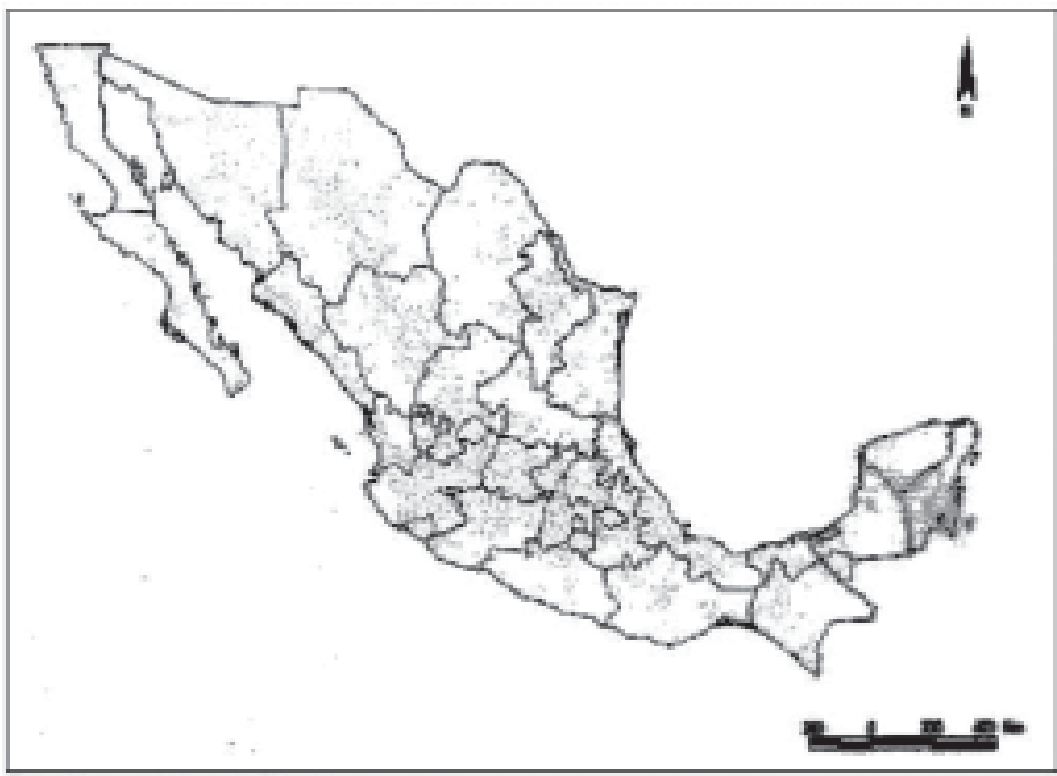

Fuente: Consejo Nacional de Población. 
Mapa IX

México: localidades con grado de marginación muy bajo

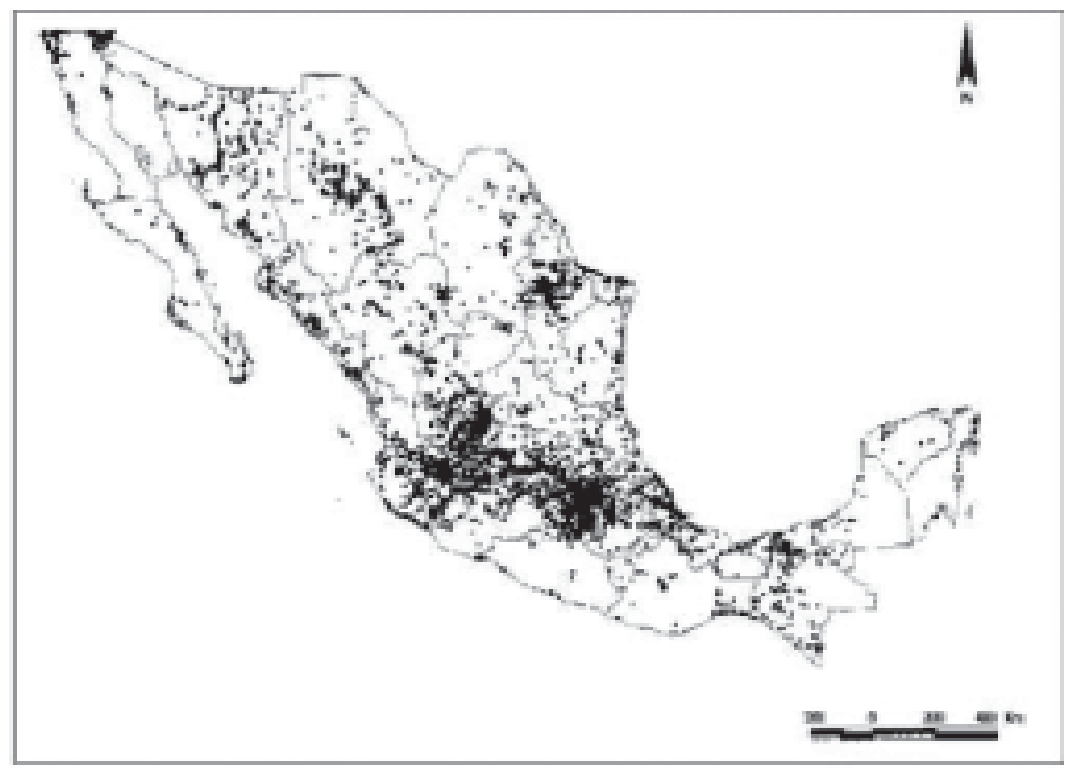

Fuente: Consejo Nacional de Población.

\section{Bibliografía}

Arroyo-Alejandre, Jesús (1993) "Migración a Estados Unidos, desarrollo de ciudades medias y la política de liberalización económica: el caso de Jalisco", en Jesús Arroyo Alejandre (comp.), Impactos regionales de la apertura comercial, perspectivas del tratado de libre comercio en Jalisco. Guadalajara, Universidad de Guadalajara, pp. 233-254.

Brenner, Neil (2003), "La formación de la ciudad global y el reescalamiento del espacio del Estado en la Europa Occidental postfordista”, en <http://www.scielo.cl/scielo.php? script $=$ sci_arttext $\&$ pid $=$ S0250-71612003008600 $001 \& \operatorname{lng}=\mathrm{es} \& \mathrm{nrm}=\mathrm{iso}>, 29(86)$, EURE, Santiago, 16 noviembre 2006, pp. 5-35.

Izunsa-Vizuet, Georgina (2005), “Transición demográfica y migraciones internas en la ciudad de México", Centro de Investigaciones Económicas, Administrativas y SocialesInstituto Politécnico Nacional, ponencia presentada en el Segundo Encuentro Internacional sobre Migraciones, del 
7 al 25 de noviembre de 2005, vía Internet, organizado por el grupo Eumed.Net de la Universidad de Málaga. Disco compacto propiedad del grupo Eumed.Net que se puede solicitar a su director, doctor Juan Carlos Martínez Coll (coll@uma.es).

Janoschka, Michael (2002), "El nuevo modelo de la ciudad latinoamericana: fragmentación y privatización”, en http:// www.scielo.cl/scielo.php?script $=$ sci_arttext $\&$ pid $=S 0250$ 71612002008500002\&lng =es\&nrm=iso, EURE, 28(85), Santiago,16 de noviembre de 2006, pp.11-20.

Maldonado-Cruz, Pedro y José del Carmen Palma Sosa (2006), Correspondencia entre el crecimiento económico y las condiciones de vida de la población, edición electrónica en www.eumed.net/libros/2006a/pmc/.

Sassen, Saskia (1998), "Ciudades en la economía global: enfoques teóricos y metodológicos”, en http://www.scielo.cl/ scielo.php?script $=$ sci_arttext $\&$ pid $=$ S0250-7161199 8007100001\&lng = es\&nrm =iso, EURE, 24(71), Santiago, 16 de noviembre de 2006, pp. 5-25.

Recibido: 21 de julio de 2006. Aceptado: 18 de enero de 2007.

Jorge Isauro Rionda Ramírez. Es doctor en estudios laborales egresado de la Universidad Autónoma Metropolitana (Unidad Iztapalapa). Actualmente es profesor-investigador de tiempo completo de la Universidad de Guanajuato adscrito al Centro de Investigaciones Humanísticas; es miembro del Sistema Nacional de Investigadores (nivel I) Conacyt. Sus línea de investigación se centra en: desarrollo regional, teoría y política económica. Entre sus publicaciones destacan: Prolegómenos en torno a la realidad social, politica y económica de México (2000), Universidad de Guanajuato, México; Historia Demográfica de Guanajuato (2005), Universidad de Guanajuato, México. 